\title{
IMMERSIONS OF SEMIANALYTIC SPACES
}

\author{
G. S. WELLS
}

\begin{abstract}
It is proved that $d: \operatorname{Imm}(V, M) \rightarrow L$ is a weak homotopy equivalence, where $\operatorname{Imm}(V, M)$ denotes the space of smooth immersions of a compact semianalytic space $V$ into a manifold $M, L$ denotes the space of continuous bundle maps, linear and injective on each fibre, from the Zariski tangent bundle of $V$ to the tangent bundle of $M$, and $d$ is the differential. This generalizes the Haefliger-Poenaru-Hirsch-Smale immersion theory for compact manifolds.
\end{abstract}

1. Locally. Let $V$ be a compact subset of $R^{n}$. $C^{\infty}(V)$ denotes the Fréchet space of smooth functions on $V$ with the Whitney topology. By a theorem of L. M. Graves (see [1] for references to Graves' work) the epimorphism $C^{\infty}\left(R^{n}\right) \rightarrow C^{\infty}(V)$ induced by restriction has a continuous right splitting $E$. For any point $p$ of $V$ let $T_{p}^{*}(V)=m_{p} / I(V)+m_{p}^{2}$, where $m_{p}$ denotes the ideal in $C^{\infty}\left(R^{n}\right)$ of smooth functions vanishing at $p$ and $I(V)$ denotes the ideal of smooth functions vanishing on $V$, and let $T_{p}(V)$, the (Zariski) tangent space of $V$ at $p$, be the dual of $T_{p}^{*}(V)$. The dimension of $V$ is $\operatorname{Max}_{p \in V}\left(\operatorname{dim} T_{p}(V)\right)$ and $T(V)=\cup_{p \in V} T_{p}(V)$, the tangent bundle of $V$, is topologised as a subspace of $T\left(R^{n}\right)$.

$f \in C^{\infty}(V, M)$ is called a (smooth) immersion of $V$ in $M$ if for each $p \in V$ there is a neighborhood $U_{p}$ such that $f^{*}: C^{\infty}(M) \rightarrow C^{\infty}\left(U_{p}\right)$ is onto. By the Malgrange preparation theorem [5] this is equivalent to saying that

$$
f^{*}: T_{f(p)}^{*}(M) \rightarrow T_{p}^{*}(V)
$$

is onto for each $p \in V$, or dually that $d f_{p}: T_{p}(V) \rightarrow T_{f(p)}(M)$, the differential of $f$ at $p$ (the dual of $f^{*}$ ), is injective for each $p \in V$, where $T_{q}(M)\left(T_{q}^{*}(M)\right.$ ) denotes the ordinary tangent (respectively cotangent) space to $M$ at $q$. The space of immersions of $V$ in $M$ will be denoted by $\operatorname{Imm}(V, M)$.

Let $x_{1}, \ldots, x_{n}$ be coordinate functions for $R^{n}$ vanishing at $p$ and let $\left\{x_{i}\right.$; $i \in I\}$ be a subcollection such that their images in $T_{p}^{*}(V)$ span this space. Then by the Malgrange preparation theorem the local ring of smooth functions on $V$ at $p$ is the image under the projection map $\pi: R^{n} \rightarrow R^{|I|}$, $\left(x_{1}, \ldots, x_{n}\right) \rightarrow\left\{x_{i} ; i \in I\right\}$ of the local ring at $\pi(p)$ of the smooth functions on $R^{|I|}$; in particular $x_{j}=f_{j}\left(x_{i}\right), j \notin I, i \in I$, for smooth locally defined functions $f_{j}$ on $R^{n}$ at $p$. The equations $x_{j}=f_{j}\left(x_{i}\right)$ define a local submanifold $U_{p}$ of $R^{n}$ at $p$ of dimension $|I|$ containing $V$ locally at $p$. If $f$ is in $\operatorname{Imm}(V, M)$ and $f^{\prime}$ extends $f$ over some neighborhood of $p$ then $f^{\prime}$ will be an embedding of

Received by the editors August 22, 1977 and, in revised form, May 16, 1978. AMS (MOS) subject classifications (1970). Primary 58D10. 
$U_{p}$ sufficiently small. $f$ can be extended to a mapping $E(f)$ defined on some neighborhood $U$ of $V$ in $R^{n}$ as follows. Let $i: M \rightarrow R^{m}$ be an embedding of $M$ in some Euclidean space, let $\pi: W \rightarrow M$ be the projection from some tubular neighbourhood $W$ of $M$ in $R^{m}$ and let $E^{\prime}: C^{\infty}\left(V, R^{m}\right) \rightarrow$ $C^{\infty}\left(R^{n}, R^{m}\right)$ be a continuous right inverse for the restriction mapping given by Graves' theorem. Then let $U=E^{\prime}(f)^{-1}(W)$ and put $E(f)=\pi \circ E^{\prime}(f)$.

2. Global. Let $V$ now be a compact semianalytic subset of $R^{n}$. $V_{i}^{\prime}=\{x \in$ $\left.V ; \operatorname{dim} T_{x}(V) \geqslant i\right\}, i=0,1, \ldots$, is closed semianalytic and the filtration $V=V_{0}^{\prime} \supset V_{1}^{\prime} \supset \cdots$ can be refined (see [10]) to a filtration by closed semianalytic sets $V=V_{0} \supset V_{1} \supset \cdots \supset V_{r+1}=\varnothing$ such that

(1) the collection of associated difference sets $\left\{W_{i}=V_{i}-V_{i+1}, i=\right.$ $0, \ldots, r\}$ is a stratification by $C^{1}$ submanifolds of $R^{n}$ satisfying the "axiom of the frontier" (Mather [7]), such that for $x \in W_{i}$ the tangent space $T_{x}(V)$ has constant dimension and contains $T_{x}\left(W_{i}\right)$, the ordinary tangent space to $W_{i}$ at $x$;

(2) the $(r+2)$-tuple $\left(R^{n}=V_{-1}, V_{0}, V_{1}, \ldots, V_{r}\right)$ can be triangulated.

In fact the filtration gives analytic $W_{i}$ but only $C^{1}$ strata are needed in what follows, and (2) follows from [4]. Only properties (1) and (2) of $V$ will be used in the rest of the paper. By a simple piecing together argument applied to the $U_{p}$ of $\S 1$ for points $p$ of $W_{i}$, for decreasing $i$, there is a collection $\left\{N_{i}\right.$; $i=0, \ldots, r\}$ of differentiable submanifolds of $R^{n}$ such that $W_{i} \subseteq N_{i}$ and

(1) $N_{i} \cap N_{j} \neq 0$ for $i>j$ only if $\bar{W}_{j} \cap W_{i} \neq \varnothing$;

(2) $N_{i}$ is diffeomorphic to the unit disk bundle of $\left(T(V) \mid W_{i}\right) / T\left(W_{i}\right)$, with $W_{i}$ corresponding to the zero cross section under the diffeomorphism;

(3) for $x \in N_{i} \cap N_{j}, i>j$, then $T_{x}\left(N_{i}\right) \supseteq T_{x}\left(N_{j}\right)$;

(4) $\bar{N}_{i}-N_{i}=\bar{W}_{i}-W_{i}$.

Let $M_{i}=\operatorname{cl}\left(N_{i}-\cup_{j>i} N_{j}\right), i=0, \ldots, r$ be the associated collection of "closure difference sets". By putting the boundaries of the $N_{i}$ into general position it can be assumed that in addition

(5) the collection of $M_{i}$ at any point $p$ of $R^{n}$ is locally differentiably equivalent to a point in a half-space flag, where a half-space flag is the collection of closure difference sets of a collection $X_{1}, X_{2}, \ldots, X_{s}$ of half-subspace of $R^{n}$ of the form

$$
\begin{aligned}
X_{j}=\left\{x_{i}=0,1 \leqslant i \leqslant n_{j}, \text { but } i \neq n_{1}, \ldots, n_{j}, x_{n_{j}}\right. & \geqslant 0\}, \\
j & =1, \ldots, s, \text { where } 0<n_{1}<n_{2} \ldots n_{s} \leqslant n+1
\end{aligned}
$$

(if $n_{s}=n+1$ the condition $x_{n+1} \geqslant 0$ is vacuous).

Let $N=\cup_{i=0}^{r} N_{i}$ and let $L(T(N), T(M))$ denote the space of continuous fibrewise linear-injective bundle maps from $T(N)$ to $T(M)$ with $\left\{N_{i}\right\}$ satisfying conditions (1) to (5) above.

Proposition. $\operatorname{Imm}(N, M)$ is weakly homotopy equivalent to $L(T(N), T(M))$ when $\operatorname{dim} N<\operatorname{dim} M$, induced by the differential. 
Proof. The handlebody approach to immersions [2] adapts immediately to manifolds with corners (as defined, say, in [6]) and we will assume the theory of immersions of these. Since each $M_{i}$ is a manifold with corners it is sufficient, following [2], to show that the restrictions

$$
\operatorname{Imm}\left(Z_{i}, M\right) \rightarrow \operatorname{Imm}\left(Z_{i+1}, M\right)
$$

and

$$
L\left(T\left(Z_{i}\right), T(M)\right) \rightarrow L\left(T\left(Z_{i+1}\right), T(M)\right), \quad i=0, \ldots, r-1,
$$

where $Z_{i}=\cup_{j>i} N_{j}$, both have the covering homotopy property with respect to $X$-parameter families of maps, where $X$ is any compact set. But an $X$-parameter homotopy can be extended to a neighbourhood of $Z_{i+1} \cap M_{i}$ in $M_{i}$ which can be assumed to be a submanifold with corners of $M_{i}$ and so the results follow from the corresponding theorems for manifolds with corners.

3. The theorem. Let $\left\{\tilde{N}_{i} ; i=0, \ldots, r\right\}$ be a fixed collection of submanifolds of $R^{n}$ as in $\S 2$ and let $C$ be the collection of all such collections $c=\left\{N_{i}\right.$, $i=0, \ldots, r\}$ with $N_{i} \subseteq \tilde{N}_{i}, i=0, \ldots, r$. For $c^{\prime}=\left\{N_{i}^{\prime}\right\}, c^{\prime \prime}=\left\{N_{i}^{\prime \prime}\right\}$ which are both in $C$ put $c^{\prime}<c^{\prime \prime}$ if $Z_{i}^{\prime \prime} \subseteq Z_{i}^{\prime}$ for all $i$ (in the notation of §2). [X,Y] denotes the set of homotopy classes of maps from $X$ to $Y$.

For $f$ in $\operatorname{Imm}(V, M)$ then $E(f)$ (in the notation of $\S 1$ ) is an immersion of $N=\cup_{i=0}^{r} N_{i}$ for $c=\left\{N_{i}\right\}$ sufficiently small and so there is an induced map, for any compact $X$,

$$
\alpha:[X, \operatorname{Imm}(V, M)] \rightarrow \lim _{c}[X, \operatorname{Imm}(N, M)],
$$

which is clearly bijective.

$$
\delta: \lim _{c}[X, \operatorname{Imm}(N, M)] \rightarrow \lim _{c}[X, L(T(N), T(M))]
$$

induced by the differential is bijective by $\S 2$. Let $D$ be the collection of $\left\{U_{i}\right.$, $i=1, \ldots, r\}$ such that $U_{i}$ is a closed relative neighbourhood of $V_{i} \bmod V_{i+1}$ in $V$ with $U_{i}-V_{i+1} \subseteq \tilde{N}_{i}$, ordered in the same way as $C$. By the neighbourhood deformation retract property of simplicial complexes the natural restriction

$$
\beta: \lim _{c}[X, L(T(N), T(M))] \rightarrow \lim _{d}\left[X, L\left(T(\tilde{N})_{d}, T(M)\right)\right]=L(X)
$$

is bijective, where $T(\tilde{N})_{d}=\cup_{i=0}^{r} T\left(\tilde{N}_{i}\right) \mid\left(U_{i}-V_{i+1}\right)$. Hence the following result holds, with $\partial=\beta \delta \alpha$.

THEOREM A. When $V$ satisfies conditions (1) and (2) of $\$ 2$ and $\operatorname{dim} V<$ $\operatorname{dim} M \partial$ is bijective (for compact $X$ ).

In order to formulate this result in ordinary homotopy theory we will find $d \in D$ such that the natural map

$$
\left[X, L(T(\tilde{N}))_{d}, T(M)\right] \rightarrow L(X)
$$

is bijective. 
4. Reformulation. If $Y$ is a subset of $X$ and $Z$ and $\pi: X \rightarrow Z$ is a continuous map with $\pi \mid Y=1_{Y}$ then the mapping cylinder of $\pi$ relative to $Y$, $M(X, Y ; \pi)$, is the quotient if the disjoint union $(X \times I) \cup Z$ under the equivalence given by $(x, 0) \sim \pi(x), x \in X$, and $(y, t) \sim y$ for $(y, t) \in Y \times$ I. We also denote by $\pi: M(X, Y ; \pi) \rightarrow Z$ the continuous map given by $\pi(x, t)=\pi(x)$ for $(x, t) \in X \times I$ and $\pi \mid Z=1_{Z}$ and let $\sigma$ and $\rho$ respectively be the continuous maps from $M(X, Y ; \pi)-Z \approx X \times I$ obtained by projection onto $X$ and $I$ respectively. $X$ will be naturally identified with $X \times 0$ in what follows and $\frac{1}{2} M(X, Y ; \pi)$ will denote the image in $M(X, Y ; \pi)$ of $X \times\left[0, \frac{1}{2}\right] \cup Z$.

If $A$ is a subcomplex of $B$ then points of the open simplicial neighbourhood $U$ of $A^{(1)}$ in $B^{(1)}$ (the first deriveds) lie on unique line segments joining points of $A$ to points of bdy $(U)$ thus inducing a projection $\pi: U \rightarrow A$, and this gives a natural identification of $U_{1}$, the closed simplicial neighbourhood of $A^{(2)}$ in $B^{(2)}$ with the mapping cylinder of $\pi / \partial U_{1}$. More generally, for the $r$-tuple $\left(K_{1}, \ldots, K_{r}\right)$ of subcomplexes of $K=K_{0}$ (in the notation of $\$ 2$ ) the second derived of $K$ gives a sequence of closed neighbourhoods $N_{i}$ of $K_{i} \bmod K_{i+1}$, $i=0, \ldots, r$ and homeomorphisms

$$
h_{i}: M_{i}=M\left(\partial N_{i}, K_{i+1} ; \pi_{i}\right) \rightarrow N_{i}
$$

for suitable $\pi_{i}: \partial N_{i} \rightarrow K_{i}$ with $\pi_{i} \mid \partial M_{i} \times 1 \approx \partial N_{i}$ equal to $1_{\partial N_{i}}$, such that (identifying each $N_{i}$ with $M_{i}$ under $h_{i}$ )

(1) $N_{i} \cap N_{j} \neq \varnothing$ for $i>j$ only if $\operatorname{cl}\left(K_{j}^{\prime}\right) \cap K_{i}^{\prime} \neq \varnothing$, where $K_{k}^{\prime}=K_{k}$ $K_{k+1}$.

(2) $N_{i} \cap N_{j}, N_{i} \cap \partial N_{j}, N_{i} \cap K_{j}$, for all $i$ and $j$, are the submapping cylinders of $N_{i}$ on $\partial N_{i} \cap N_{j}, \partial N_{i} \cap N_{j}$ and $\partial N_{i} \cap K_{j}$ respectively.

(3) $\pi_{i} \pi_{j}=\pi_{i}$ for $i>j ; \sigma_{i} \sigma_{j}=\sigma_{j} \sigma_{i}$ for $i>j$ on $\left(N_{i} \cap N_{j}\right)-K_{j}$.

(4) $\rho_{j} \sigma_{i}=\rho_{j}$ on $\left(N_{i} \cap N_{j}\right)-K_{i}$.

A shrinking isotopy of $K$ is an isotopy $f_{t}, t \in I$, of $K$ which is the composition of continuous isotopies $f_{i, l}, i=0, \ldots, r$ such that $f_{i, t} \mid\left(K-N_{i}\right) \cup$ $K_{i}$ is the identity and $f_{i, t}(x, s)$ is of the form $\left(x, \phi_{s}(t)\right)$ for a monotone decreasing function $\phi_{s}$, for $(x, s)$ in $\partial N_{i} \times(0,1] \approx N_{i}-K_{i}$. Using shrinking isotopies it can be assumed that $U_{i} \subseteq \tilde{N}_{i}$ where $U_{i}=\frac{1}{2} N_{i}$ and then it is a simple exercise in the covering homotopy theorem for bundles to show that $d=\left\{U_{i} ; i=0, \ldots, r\right\}$ satisfies the condition at the end of $\S 3$ and so Theorem $\mathbf{A}$ implies the following result.

THEOREM B. $\operatorname{Imm}(V, M) \rightarrow L\left(T(\tilde{N})_{d}, T(M)\right)$, induced by the differential, is a weak homotopy equivalence under the conditions of Theorem A.

5. Smale theory. Let $L$ be the space of continuous fibre maps, linear and injective on fibres, from $T(V)$ to $T(M)$ and let $L^{\prime}=L\left(T(\tilde{N})_{d}, T(M)\right.$ ). Choose homotopies $\gamma_{i}$ of id: $N_{i} \rightarrow N_{i}, i=0, \ldots, r$, such that $\gamma_{i 0}=\mathrm{id}, \gamma_{i t} \mid \partial N_{i}$ $=\mathrm{id}, \pi_{i} \gamma_{i t}=\pi_{i}, \rho_{i} \gamma_{i t}(x)$ decreases with $t$ for $x$ in $N_{i}$, and $\gamma_{i 1}^{-1}\left(K_{i}\right)=U_{i}$. 
Extend $\gamma_{i}$ to $K$ by the identity outside $K_{i}$, let $\Gamma$ be the (homotopy) composition $\gamma_{0} \cdot \gamma_{1} \cdots \gamma_{r}$ and let

$$
N_{i}^{\prime}=N_{i}-\bigcup_{j>i} U_{j}, \quad U_{i}^{\prime}=N_{i}^{\prime} \cap U_{i} \text {. }
$$

By the covering homotopy property (and induction on $i$ ) there are continuous families of bundle isomorphisms, "covering" $\Gamma_{t}, \tau_{i t}: T\left(\tilde{N}_{i}\right) \mid N_{i}^{\prime} \rightarrow$ $\Gamma_{t}^{*}\left(T\left(\tilde{N}_{i}\right)\right) \mid N_{i}^{\prime}$ such that $\tau_{i 0}=$ id and

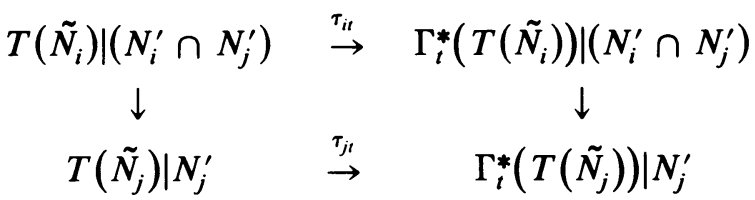

commutes for $j>i$, where the vertical maps are induced by the inclusion $T\left(\tilde{N}_{i}\right) \subseteq T\left(\tilde{N}_{j}\right)$ on their common domain. Let $r: L^{\prime} \rightarrow L$ be the natural "restriction"; a homotopy inverse $\gamma$ for $r$ will be found. For $f$ in $L$ let $\gamma f \mid\left(T\left(\tilde{N}_{i}\right) \mid U_{i}^{\prime}\right)$ be given by the composition of

$$
\tau_{i 1}: T\left(\tilde{N}_{i}\right) \mid U_{i}^{\prime} \rightarrow \Gamma_{1}^{*}\left(T\left(\tilde{N}_{i}\right) \mid U_{i}^{\prime}\right)
$$

with the natural map into $T\left(\tilde{N}_{i}\right)$ followed by $f \mid\left(\left.T\left(\tilde{N}_{i}\right)\right|_{K_{i}^{\prime}}\right.$ ) (note that $T\left(\tilde{N}_{i}\right)$ and $T(V)$ coincide over $K_{i}^{\prime}$ ). Since $\Gamma$ is homotopic as a map of $\left(V_{0}, V_{1}, \ldots, V_{r}\right)$ into itself to the identity, $r \gamma$ is homotopic to $\mathrm{id}_{L}$, and using $\left\{\tau_{i t}, t \in I\right\}$ it follows easily that $\gamma r$ is homotopic to $\mathrm{id}_{L^{\prime}}$. Then by Theorem B we have the main theorem.

THEOREM. The differential $d: \operatorname{Imm}(V, M) \rightarrow L$ is a weak homotopy equivalence.

\section{REFERENCES}

1. N. Dunford and J. T. Schwartz, Linear operators. I. General theory, Interscience, New York, 1958.

2. A. Haefliger and V. Poenaru, La classification des immersions combinatoires, Inst. Hautes Etudes Sci. Publ. Math. 23 (1964), 75-91.

3. M. Hirsch, Immersions of manifolds, Trans. Amer. Math. Soc. 93 (1959), 242-276.

4. S. Lojasiewicz, Triangulation of semi-analytic sets, Ann. Scuola Norm. Sup. Pisa (3) 18 (1964), 449-474.

5. B. Malgrange, Ideals of differentiable functions, Oxford Univ. Press, London, 1967.

6. J. N. Mather, Stability of $C^{\infty}$ mappings. II, Ann. of Math. (2) 89 (1969), 254-291.

7. Stratifications and mappings, Dynamical Systems, Academic Press, New York, 1973.

8. G. S. Wells, Spaces of smooth functions on analytic sets, Bull. Amer. Math. Soc. 83 (1977), 276-278.

9. Immersions of $G$ manifolds, $G$ finite, Bull. Amer. Math. Soc. 74 (1968), 130-132.

10. $\quad$ A geometric splitting and the $C^{\infty}$ stability of maps from semianalytic spaces, Quaestiones Math. 2 (1977), 383-399.

Department of Mathematics, University of the Witwatersrand, 1 Jan SMuts Ave., JohanNesburg, 2001, Republic OF SOUTH AFrica 